\title{
Mass Transfer in Infrared Drying of Gel-Coated Seeds
}

\author{
Antônio M. Barbosa Neto ${ }^{1}$, Luanda G. Marques ${ }^{1}$, Manoel M. Prado ${ }^{1 *}$, Dermeval J. M. Sartori ${ }^{2}$ \\ ${ }^{1}$ Department of Chemical Engineering, Federal University of Sergipe, São Cristóvão, Brazil \\ ${ }^{2}$ Department of Chemical Engineering, Federal University of São Carlos, São Carlos, Brazil \\ Email: "manoelprado@ufs.br
}

Received December 8, 2013; revised January 8, 2014; accepted January 14, 2014

Copyright (C) 2014 Antônio M. Barbosa Neto et al. This is an open access article distributed under the Creative Commons Attribution License, which permits unrestricted use, distribution, and reproduction in any medium, provided the original work is properly cited. In accordance of the Creative Commons Attribution License all Copyrights (C) 2014 are reserved for SCIRP and the owner of the intellectual property Antônio M. Barbosa Neto et al. All Copyright @ 2014 are guarded by law and by SCIRP as a guardian.

\section{ABSTRACT}

In order to contribute for a better understanding of mass transfer in drying of shrinking particles, in this study shrinkage and drying characteristics of sorghum seeds encapsulated into gel-based polymeric matrix were experimentally determined by using infrared (IR) radiation. IR drying of gel coated seeds was carried out at three different temperatures $\left(65^{\circ} \mathrm{C}, 80^{\circ} \mathrm{C}\right.$ and $\left.93^{\circ} \mathrm{C}\right)$. The shrinkage of the individual particles during drying was quantified by means of the volume and surface area changes evaluated from geometric measurements. The product quality was evaluated in terms of the changes of particle density and percent of cracks in gel coating incurred during drying. Surface area and volume of the gel-seed system decreased about $65 \%$ and $80 \%$ until the end of the process, respectively, stressing the need to take into account the surface area changes to calculate water flux density as function of moisture content and obtain an accurate interpretation of the drying mechanisms well as to include the volume shrinkage in mass transfer models to determine reliable values of moisture diffusivity. The IR drying behavior of gel-coated seeds was then characterized by the presence of three drying periods: heating up, constant moisture flux and falling moisture flux. Accelerated drying of gel coated seeds was obtained by applying higher IR radiation intensities. The effect of IR source temperature on the particle shrinkage was more pronounced at the constant moisture flux period and practically negligible at the decreasing moisture flux period. Neglecting shrinkage of individual coated-seeds during IR drying led to an erroneous absence of constant flux period and overestimation of the mass transfer by diffusion. Apparent density of the particles was greater at lowtemperature IR drying than at high-temperature IR drying. Coated particles keep their original geometry, but a significant cracking of gel coating was observed at rapid drying rate conditions.

\section{KEYWORDS}

\section{Gel Coating; Shrinking Particles; Infrared Drying; Mass Transfer}

\section{Introduction}

Sorghum [Sorghum bicolor (L.) Moench] is one of the main agricultural crops in the world scenery. It is cultivated in Brazil predominantly for the production of grain and forage, but a growing interest is being focused on the potential of sorghum to produce bioethanol feedstocks [1]. Unlike sugar cane, sorghum is grown from seed and has a much shorter growing season of around 120 to 130 days. It is also capable of growing on marginal lands where other crops do not thrive.

Breeding programs for new varieties of sorghum suited to semi arid tropics are under development. How-

${ }^{*}$ Corresponding author. ever, in the semiarid regions of the Brazilian Northeast, water and salinity stresses are primary limiting environmental conditions which restrict successful establishment of crops. Seed germination may be significantly reduced and delayed by the decreasing rate of water absorption, when drought stress and soil salinity are high or when saline water is used for irrigation [2].

Among the numerous seed treatment methods, the coating with hydrocolloids due to their hydrophilic characteristics can be viewed as a promising technique to be performed on sorghum seeds, in order to overcome constraints to seed germination, as the water stress in soil, a common problem in areas of Brazilian Northeast, with 
low rainfall [3]. Polymer-based coating may also reduce the leaching of inhibitors from the seed coverings as well as restrict salts and oxygen diffusion to the embryo [4]. In addition, polymer coating can regulate moisture sorption during storage, providing protection against insect infestation and microbial attack and improving seed conservation. Hydrocolloid-based polymeric matrices such as agar and alginate gels have been successfully employed for encapsulating seeds of many crops, improving their germination and emergence $[5,6]$.

Drying is an essential operation in seed coating since this step ensures moisture removal from the coating, promoting the gel adherence to the seed surface and preventing deterioration due to molds infestation. According to Kajtna et al. [7], the drying process does not improve the adhesion properties of the coating, which are dependent on the polymer properties. However, improperly chosen drying conditions may cause polymer degradation and worsen the adhesion properties, leading to losses in coated seed quality.

The increased awareness about environmental degradation has motivated the application of alternative sources of clean energy in drying operations [8]. Within this context, drying using infrared (IR) radiation appears as an interesting method to intensify heat and mass transfer rates, to minimize energy consumption and to preserve quality of coated seeds. The layer-layer drying of the material by applying IR radiation can prevent transfer of soluble substances beyond the product surface and formation of surface films, and the relatively high rate of drying suppresses oxidation and prevent heatsensitive substances being lost from the product during drying. Preserving the integrity of the gel coating structure allows maintaining its ability to imbibe water, which is an essential quality attribute of coated seeds in the germination process.

The application of IR radiation for drying purposes in chemical and agro-food industries has become widely widespread, due to its intrinsically useful characteristics such as higher energy efficiency, shorter drying time, versatility, simplicity of equipment, fast response of heating and drying, easy incorporation to any existing dryer and low capital cost [9]. A lot of work has been devoted to the research on IR drying of various materials, such as: paper [10], polymers [7], pharmaceutical films [11], paints and pigments [12,13], hydrous ferrous sulphate [14], and agricultural products [15-18]. However, there is still no information available in the literature regarding the use of IR radiation for drying systems composed by seeds coated by gel-based polymeric matrices.

The infrared drying of gel-coated particles is a challenging assignment due to the complex relationship between the transport phenomena and the shrinkage characteristics, which are directly related to quality attributes, such as density, porosity, sorption characteristics, crust and cracks [19]. Due to the highly deformable polymeric structure of gel coating, gel coated seeds undergo changes in their original dimensions and shape during moisture removal, modifying particle structural properties as well as the heat and mass exchange area, thus affecting the drying rate. The complexity increases as the extent of shrinkage is also process dependent. That is the result of the moisture gradient in the product, which, in turn, induces stresses and, thus, mechanical deformation [19].

Thus, any attempt to raise the shrinkage phenomenon to a higher level of understanding must address the structural properties of the material such as density, porosity, specific area and surface cracking.

The changes in shape, dimensions and solid structure of deformable particles yield to a particular system where the available coefficients of mass transfer are not suitable to reproduce moisture loss during drying of the material. Thus, any attempt to accurately interpret the mass transfer phenomenon during drying of shrinking particles like gel coated seeds requires an experimental investigation to obtain reliable values of effective moisture diffusivity $\left(D_{\text {eff }}\right)$, which should take into account the reduction in the distance required for the movement of water molecules. However, few works have been carried out on the effects of the shrinkage phenomenon on $D_{\text {eff }}$, even less on $D_{\text {eff }}$ of gel coated seeds.

The quantification of the particle shrinkage and its dependence on process variables is essential to improve the understanding of drying kinetics and hence to provide more realistic information on the mechanism of moisture transport, the influence of operating conditions on the drying behavior as well as for selection of optimal drying conditions for seed quality control $[20,21]$.

The objectives of this work were, therefore, to determine the shrinkage and drying characteristics of agar gelcoated sorghum seeds subjected to infrared radiation as well to evaluate the influence of particle shrinkage on the effective moisture diffusivity. The effects of IR radiation intensity on the percent of cracks in gel coating were also examined.

\section{Materials and Methods}

\subsection{Materials}

In this study, sorghum (Sorghum bicolor L. Moenche) seeds from Embrapa BRS-330 variety were used. This early duration variety is tannin-free and is characterized by high protein content. It was grown at Experimental Station of Embrapa Maize and Sorghum, Sete Lagoas/ MG.

The materials used for forming coating matrices included agar agar (Acumedia Manufactures, Maryland, 
USA) as gelling agent, sorbitol (Merck) as plasticizing agent, starch powder (Vetec Química Fina Ltda) as binder, and water as solvent. Filler agents of the coating, as cellulose, talc and titanium dioxid, were generously provided by Minérios Ouro Branco Ltda, São Paulo, Brazil.

\subsection{Preparation of Materials}

Sorghum seeds were received from Embrapa Maize and sorghum with a moisture content of 0.126 wet basis (d.b.), at room temperature, and free from broken grains and foreign material. Seed size distribution was firstly determined from sieve analysis. Medium seeds (sieve diameter between 3.6 and $4 \mathrm{~mm}$ ) were found to represent about $80 \%$ population in the lot. Thus they were chosen for the study, in order to ensure uniform particle size.

The coating mixture was prepared in accordance with the Committee on Food Chemicals Codex [22]. It had the following formulation: $1.5 \%$ agar, $0.5 \%$ sorbitol, $1.5 \%$ starch, $4 \%$ de cellulose, 0.5\% titanium dioxid and 92\% water. This formulation provided the best adherence of the coating mixture to the seed surface.

\subsection{Coating Methodology}

Application of the polymeric mixtures in rectangular moulds containing the seeds was the adopted technique for entrapping the sorghum seeds within gel matrices. The beaker containing the polymeric mixture was immersed in a thermal bath at $50^{\circ} \mathrm{C}$ to maintain the material in gum phase. After poured into the moulds the coating mixtures were allowed to gel at room temperature for $2 \mathrm{~h}$ to enable high gel strength prior to removing gel-seed system from the moulds. This resulted in the formation of encapsulated seeds, rectangular in shape $6 \mathrm{~mm} \times 12$ $\mathrm{mm} \times 18 \mathrm{~mm}$ (thickness $\times$ width $\times$ length).

\subsection{Drying of Gel-Seed System}

The experimental set-up developed to study the single layer drying of gel coated sorghum seeds using infrared radiation is shown schematically in Figure 1 . The experimental unit consists of a drying chamber equipped with a $250 \mathrm{~W}$ incandescent lamp. The drying chamber of 200 $\times 200 \times 300 \mathrm{~mm}$ was made from wood sheet of $10 \mathrm{~mm}$ thickness. The chamber walls were isolated and covered with an aluminum foil. The temperature of the IR source was regulated using a temperature controller. The distance between the infrared heating source and the monolayer of particles was set at $17 \mathrm{~cm}$. Drying tests were conducted with IR source temperatures of $65^{\circ} \mathrm{C}, 80^{\circ} \mathrm{C}$ and $93^{\circ} \mathrm{C}$.

Two experiments groups were carried out. The aim of the first group was to determine the drying kinetics of the gel-coated seeds at different IR radiation intensities in terms of the moisture content and temperature evolution.

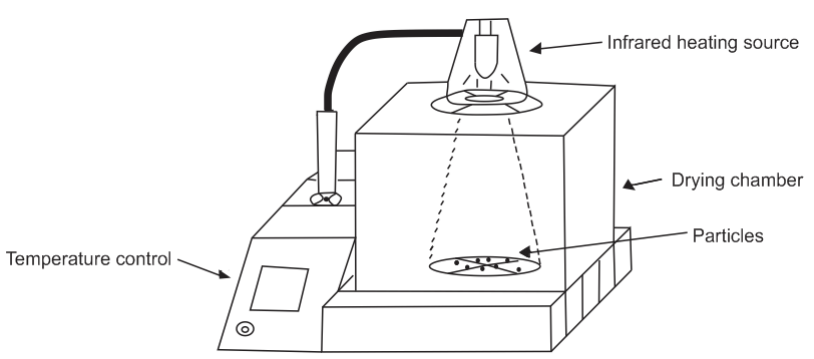

Figure 1. Schematic diagram of the experimental set-up.

The second group had as objective to identify and quantify the shrinkage of the gel-coated particles as a function of moisture content and IR drying conditions.

The dryer was run empty for about $0.5 \mathrm{~h}$ to attain a steady state in respect of the pre-set operating conditions before each drying test. About $22 \mathrm{~g}$ of sample were used at each experiment. Seeds were uniformly spread on the aluminum tray (130 mm diameter) in a mono layer. Each experiment and subsequent analyses were performed in triplicate.

To determine the drying kinetics curves the seed samples were removed from the equipment at regular intervals along the total drying time and their weight measured in a digital balance (Sartorius, CP224S, Germany) with $10^{-4} \mathrm{~g}$ accuracy. All weighting processes were completed in $15 \mathrm{~s}$ during the drying process. Moisture content at a given time was calculated by the ratio between the mass of evaporated water and the dry mass, which was determined at the end of each experiment by the oven method at $105^{\circ} \mathrm{C} \pm 3^{\circ} \mathrm{C}$ for 24 hours. The surface temperature of the particles was measured at different drying times by using an optical pyrometer.

Coated seeds samples were subjected to IR radiation for different periods to achieve the desired moisture content, with previously drying kinetics curves facilitating determination of the drying time. On attaining the predetermined drying time, the coated seeds were taken from the dryer and their dimensions were measured using a digital micrometer (Pantec, Model 13101-25, accuracy of $10^{-3} \mathrm{~mm}$ ). The formation of cracks in the coating structure during drying was quantified by image analysis. Percent of breakage was then used as quality attribute of the coated seeds.

\subsection{Analysis of Drying Kinetics and Particle Shrinkage Data}

The experimental data sets from the different drying tests were initially expressed as moisture content $(X)$ versus time $(t)$. The moisture contents values were also converted into the dimensionless moisture ratios $(X R)$, defined as the ratio of the free water still to be removed at any time to the total free water initially available, and expressed as: 


$$
X R=\frac{\bar{X}-X_{e}}{X_{0}-X_{e}} .
$$

where $\bar{X}$ is the average moisture content at a given time, $X_{0}$ and $X_{e}$ are the initial and equilibrium moisture content of coated particles, respectively. All at dry basis. For IR drying data the value of $X_{e}$ in Equation (1) was set to zero since prolonged exposure of agricultural material to infrared radiation will eventually cause a complete moisture removal [23].

The shrinkage of gel coated seeds during single layer drying was quantified from the changes in volume and surface area of individual particles, which were calculated from the dimensions taken by the digital micrometer. Reduced dimensional changes in both volume $\left(V_{p} / V_{p 0}\right)$ and area $\left(A_{p} / A_{p 0}\right)$ with respect to the moisture content constituted the so-called shrinkage curves. It must be pointed out that the shrinkage analysis, based on volume and surface area changes from the geometric measurements with the aid of a digital micrometer, was sufficiently accurate since gel coated seeds keep its original shape throughout the whole drying process.

The water flux density, averaged over the entire exchange surface, was then calculated at a given time or moisture content using the following equation:

$$
N_{w}=-\frac{m_{d s}}{A_{p}} \frac{\mathrm{d} \bar{X}}{\mathrm{~d} t}
$$

where $N_{w}$ is the mass flux density $\left(\mathrm{kg} \cdot \mathrm{m}^{-2} \cdot \mathrm{min}^{-1}\right), m_{d s}$ is the weight of the dry solids $(\mathrm{kg})$, and $A_{p}$ is the surface area of the particles exposed to IR radiation $\left(\mathrm{m}^{2}\right)$. The drying rate per mass unit of dry solid $(-\mathrm{d} \bar{X} / \mathrm{d} t)$ was determined from the drying curves by numerical derivation.

The mass transfer in IR drying of gel-coated seeds was analyzed in terms of effective diffusivity, which was determined at each IR source temperature by applying diffusive model to describe the drying kinetics under the consideration of both negligible and significant shrinkage.

Non-steady mass transfer equation based on Fick's second law for the diffusion of water during drying is expressed as

$$
\frac{\partial\left(\rho_{d} X\right)}{\partial t}=\nabla \cdot\left[D_{e f} \nabla\left(\rho_{d} X\right)\right]
$$

where $D_{e f}$ is the effective diffusivity, $\rho_{d}$ is the local concentration of dry solid (kg dry solid per volume of the moist material) that varies with moisture content, because of shrinkage and $X$ is the local moisture content (dry basis).

Assuming one-dimensional moisture transfer with negligible shrinkage and considering that the process is isothermal, initial moisture distribution is uniform, effective water diffusivity into material is constant, and equili- brium conditions at the particle surface, the analytical solution for rectangular geometry, if only the first term is considered, can be approximated to the form [24]:

as

$$
X R=\frac{\bar{X}-X_{e}}{X_{0}-X_{e}}=\frac{8}{\pi^{2}} \exp \left(-\frac{\pi^{2} D_{\text {eff }}}{L_{0}^{2}} t\right)
$$

where $L_{0}$ is the slab thickness and $D_{\text {eff }}$ is the effective moisture diffusivity without considering the particle shrinkage.

In order to include the shrinkage effects, substituting the density of dry solid $\left(\rho_{d}=m_{d} / V_{p}\right)$, Equation (3) for constant mass of dry solid could be expressed as $[20,25$, 26]:

$$
\frac{\partial\left(X / V_{p}\right)}{\partial t}=\nabla \cdot\left[D_{e f} \nabla\left(X / V_{p}\right)\right]
$$

where $V_{p}$ is the particle volume. Substituting $Y=X / V_{p}$, the following equation is obtained:

$$
\frac{\partial Y}{\partial t}=\nabla \cdot\left(D_{e f f}^{*} \nabla Y\right)
$$

For one-dimension moisture transfer in conjunction with the following initial and boundary conditions

$$
\begin{gathered}
t=0, Y=X_{0} / V_{0} \\
t>0, z=0, \frac{\partial Y}{\partial z}=0 \\
t>0, z=L, Y=X_{e q} / V_{e q}
\end{gathered}
$$

a solution similar to Equation (11) is obtained:

$$
Y R=\frac{\bar{Y}-Y_{e}}{Y_{0}-Y_{e}}=\frac{X / V-X_{e} / V_{e}}{X_{0} / V_{0}-X_{e} / V_{e}}=\frac{6}{\pi^{2}} \exp \left(-\frac{\pi^{2} D_{e f f}^{*}}{L^{2}} t\right) \text { (10) }
$$

where $V_{p}$ is the sample volume at a given time, $V_{p 0}$ is the initial volume and $V_{p e}$ is the sample volume on attaining the equilibrium moisture, $D_{\text {eff }}^{*}$ is the effective moisture diffusivity of shrinking particle and $L$ is the time averaged thickness during drying.

Equations (4) and (10) are valid only for describing the falling rate period when the drying process is controlled by internal moisture diffusion and moisture content is below the critical value. Therefore, the water diffusivity into gel-coated seeds undergoing IR drying was estimated from these equations by setting the initial moisture content to the critical value $X_{c r}$ and by setting the drying time to zero when the mean moisture content of the sample reaches that critical moisture content. Experimental data of $X R$ and $Y R$ were then fitted to the Equations (4) and (10), respectively. $D_{\text {eff }}$ and $D_{\text {eff }}^{*}$-values were estimated by applying a non-linear regression procedure using STATISTICA ${ }^{\circledR}$ software. The estimation method was based on the well-established Levenberg-Marquardt 
algorithm [27]. The statistical criteria used to evaluate the goodness-of-fit of Equations (4) and (10) were the coefficient of determination $\left(R^{2}\right)$ and the root mean square error (RMSE). RMSE was calculated from the predicted and the experimental $X R$ values as follows:

$$
\mathrm{RMSE}=\sqrt{\frac{1}{n} \sum_{i=1}^{n}\left(X R_{\text {pred }, i}-X R_{\text {exp }, i}\right)^{2}}
$$

where $n$ is the number of data points.

The dependency of effective diffusivity on temperature was investigated by plotting the obtained values against product temperature. The trends were visually inspected and appropriate model was fitted to the data.

\section{Results and Discussion}

\subsection{Shrinkage Characteristics}

Experimental shrinkage data of gel coated seeds, expressed by $A_{p} / A_{p 0}$ and $V_{p} / V_{p 0}$ ratios, were plotted against the dimensionless moisture content at different IR radiation source temperatures and are presented in Figures 2 and 3 , respectively.

A steep rise in area and volume reduction of coatedseeds samples was observed at the early and intermediate drying stages. On attaining a critical $X R$-value (around 0.2 ), there is a decrease in slope of shrinkage curves and both the area and volume contraction in particles increases slowly. This change in shrinkage behavior is due to modifications in the proportion of external and internal resistances to moisture transfer [28], which are caused by changes in the mechanism of mass transfer, what will be discussed in the following section. In the final stage of drying internal resistances control the mass transport and the migration of internal moisture is not uniform, so that it does not compensate the evaporation rate from the par-

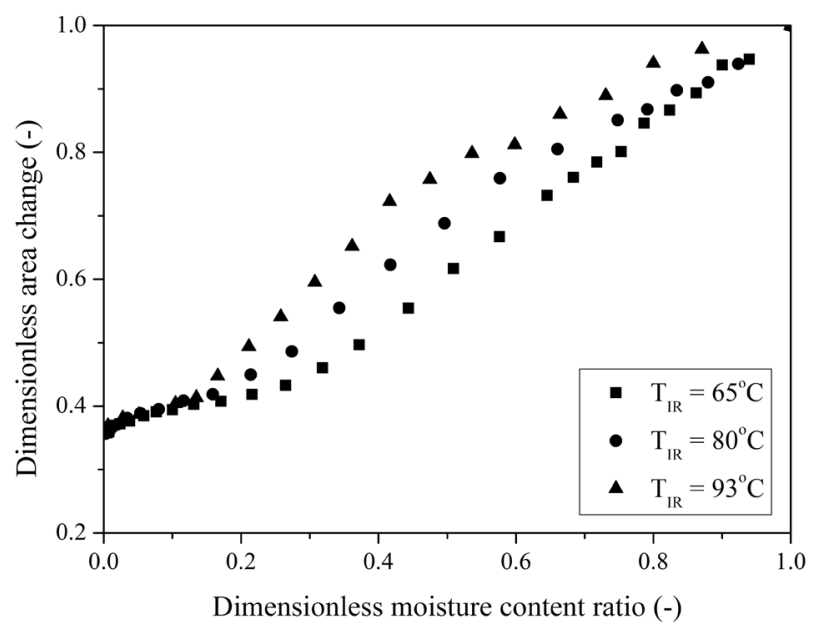

Figure 2. Dimensionless area change $\left(A_{p} / A_{p 0}\right)$ of gel-coated seeds as a function of dimensionless moisture content $(X R)$ during IR drying at different temperatures.

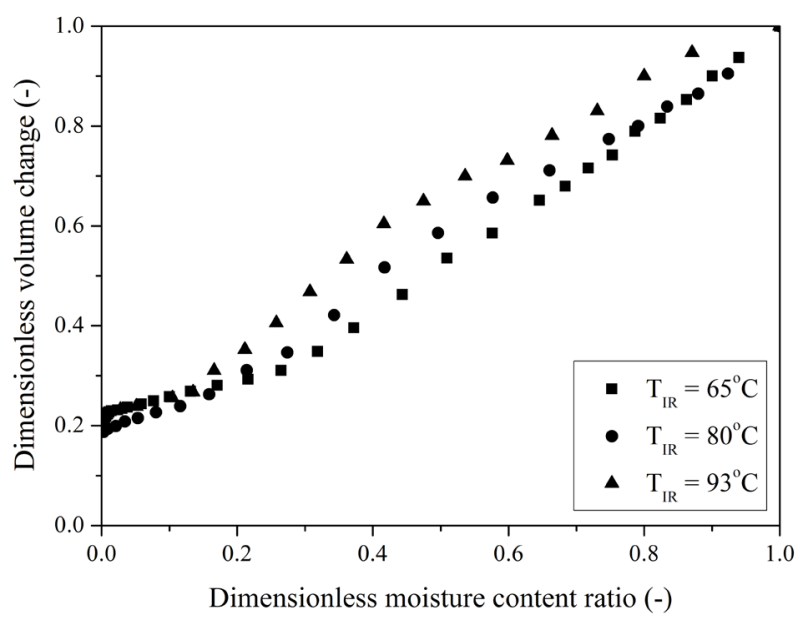

Figure 3. Dimensionless volume change $\left(V_{p} / V_{p 0}\right)$ of gelcoated seeds as a function of dimensionless moisture content $(X R)$ during IR drying at different temperatures.

ticle surface. Formation of a rigid crust in the gel coating may then be induced, limiting the particle volume, thus hindering subsequent shrinkage. This may also be attributed to the decrease at low moisture contents in the mobility of the polymeric matrix coating the seeds [29].

Particle shrinkage was found to increase with decrease in temperature of IR source, but this effect was observed to be more pronounced at the intermediate stage, until $X R$ has reached the critical value. For example, at a $X R$-value of 0.37, the reductions in both particle area (Figure 2) and volume (Figure 3 ) at $65^{\circ} \mathrm{C}$ were nearly $23 \%$ and $25 \%$ higher than that for the seeds dried at $93^{\circ} \mathrm{C}$. Exposing the particles under low drying temperatures results in low drying rates, so that external resistance controls the mass transfer, moisture profiles into the particles are uniform and stresses are minimum [28]. This contributes for the shrinkage phenomenon.

Below the $X R$ of about 0.2, the deviation within the experimental data (Figures 2 and 3) obtained under different IR-drying temperatures was smaller than the measurement uncertainties. This indicates that for the final stage of drying the temperature had a negligible effect on the shrinkage of gel coated seeds, so that both volume and surface area changes can be correlated with only the averaged moisture content of the dehydrated seeds.

At the end of IR-drying the reductions in volume and surface area of gel coated seeds for the investigated temperature range were high, approximately $80 \% \pm 2 \%$ and $64 \% \pm 1 \%$, respectively, stressing the need to take into account the surface area changes to the calculation of the water flux density as well as to include the shrinkage in the mass transfer models.

\subsection{Drying Characteristics}

Typical results of surface temperature and water flux 
density as a function of time, calculated with and without consideration of reduction in particle area exposed to IR radiation are shown in Figure 4.

Because of the high initial water content of gel-coated seeds as well as of the deformable coating structure, the course of IR drying was accompanied by both shrinking surface area and volume as discussed previously. As a result, there is a large area to volume ratio for heat and mass transfer, facilitating the moisture transport, thus leading to higher values of water flux density. On the other hand, if the reduction in mass exchange area is neglected lower values for moisture flux density are obtained and it decreases even before the completion of the first stage of drying, although free water remains available on the surface [30]. It is evident from Figure 4 that on considering constant mass transfer area no constantflux period was observed, providing a misleading interpretation of the drying mechanisms.

It should be pointed out that the presence of a constant flux period has been rarely noticed in the most of studies on IR drying [7,9,14,17], probably because mass exchange area during drying are generally not considered [31].

The analysis of drying kinetics in terms of the evolution in surface particle temperature in conjunction with the water flux density indicates that the drying behavior of gel-coated seeds exposed to IR radiation is characterized by the presence of three periods of drying: heating up, constant flux and one falling flux period.

At the beginning of drying process, under all the operating conditions, there was a warming-up period, during which both the water flux density and the temperature increase. This initial stage represents a transition period corresponding to non-isothermal conditions. The heating period was followed by a constant flux period, in which the heat and mass flux densities are equal. During this

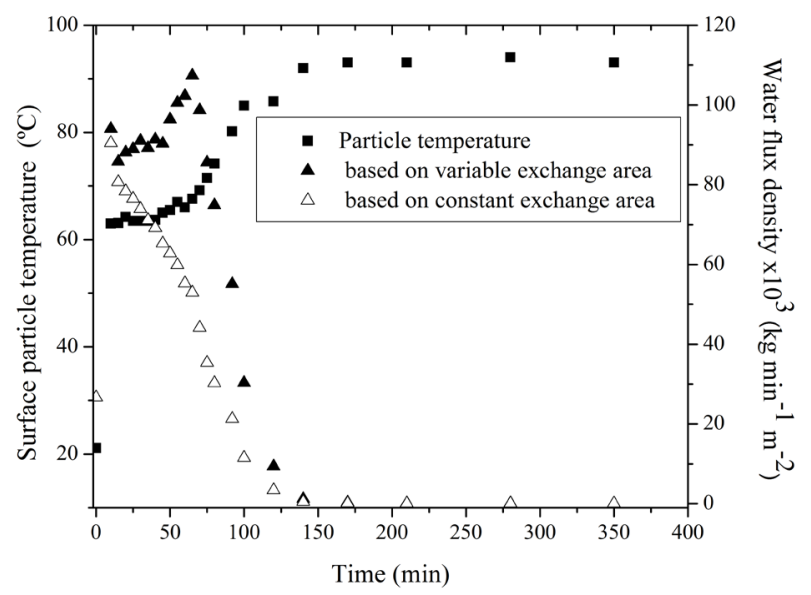

Figure 4. Surface temperature and water flux density of coated particles as a function of time during IR drying at $93^{\circ} \mathrm{C}$. period migration of internal moisture compensates the evaporation rate from the particle surface, maintaining this surface saturated with a moisture film. As a consequence, all infrared energy is used as latent heat to vaporize the surface moisture in gel-seed system and drying occurs under a constant saturation temperature, as evidenced by the temperature plateau in Figure 4. From the critical moisture critical, the aforementioned moisture compensation ceases and part of the radiant energy is used in the form of sensible heat, causing an increase in solid temperature, while the moisture flux density decreases with reduction in the moisture content, thus characterizing the falling flux period, which is mainly controlled by moisture diffusion within the particles. This is a complex mechanism involving water in both liquid and vapor states, which is commonly characterized by a socalled effective diffusivity [24].

The changes in drying behavior presented in Figure 4 also provide evidences on the reduction in IR absorption capability as the moisture of the seeds was removed. High initial moisture content of the gel-coated seeds contributed for higher absorption of radiation energy at the early stage of drying, resulting in a raised heat content of water inside the seed. The water tends to vigorously flash out by evaporation, thus taking the latent heat of vaporization from the seed, consequently increasing the drying rate [17]. During the constant rate period, when internal moisture migration compensates the evaporation of moisture from the seed surface, mass and heat transfer rates are equal. At the falling rate period, with the particle surface drying out, heat penetration through the dried layer is decreased, thus reducing the drying rate [32].

Typical results of moisture flux density versus moisture content for gel-coated seeds samples dried using infrared radiation at different heating source temperatures are shown in Figure 5. As expected, an increase in IR

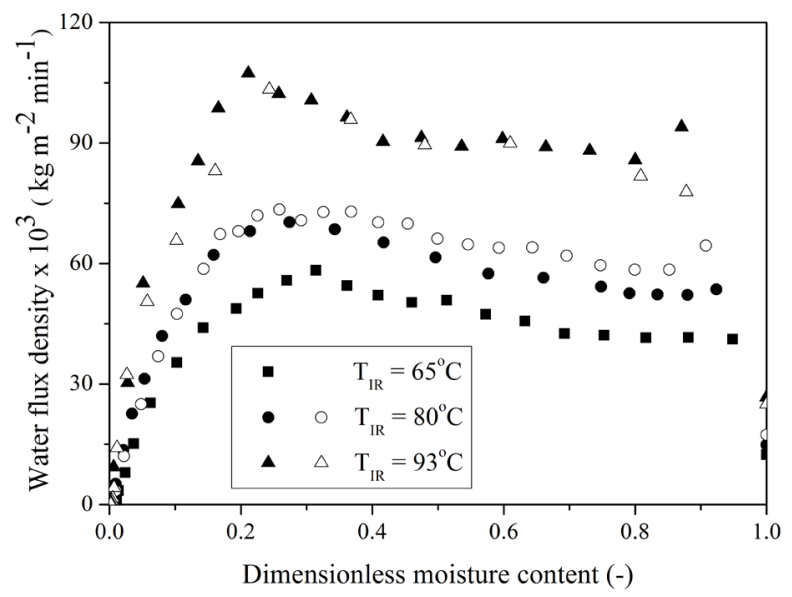

Figure 5. Moisture flux density versus moisture content during IR drying of gel coated seeds at different IR source temperatures. 
source temperature resulted in an enhancement in the overall water flux density and thus a reduced total drying time. This can be attributed to a rapid increase in particle temperature, caused by a higher intensity of radiant energy applied, resulting into an increase in the water vapor pressure inside the product and thus in higher drying flux densities [33].

An increase in drying rates with an increase in IR radiation power or intensity has commonly been reported in the literature [7-18].

Table 1 summarizes the values of moisture flux density during the constant flux period $\left(N_{w, c}\right)$, critical moisture content $\left(X_{c r}\right)$, and critical drying time $\left(t_{c r}\right)$ for all IR source temperatures $\left(T_{\mathrm{IR}}\right)$ investigated in this study.

As expected, an increase in IR source temperature resulted in a reduction of the constant flux period. However, the moisture content at which the transition between constant and falling flux periods occurred was found to be practically independent on drying conditions.

In addition, based on the critical values of XR reported, it can be stated from Figures 2 and 3 that shrinkage of particles has occurred mainly in the constant flux period.

The constant flux period was then represented by a coefficient of mass transfer $\left(k_{c}\right)$, whose relationship with the IR source temperature is expressed as:

$$
N_{w, c}=k_{c}=2.14 \times 10^{-5} T^{1.859}
$$

In what follows, the analysis of the decreasing flux period based on the application of the diffusive model is presented, aiming to evaluate the influence of particle shrinkage on the estimation of effective moisture diffusivity.

Table 1. Summary of $\boldsymbol{t}_{c r}, X_{c r}$ and $N_{w, c}$ data for single layer IR drying of gel-coated sorghum seeds.

\begin{tabular}{ccccc}
\hline $\begin{array}{c}T_{I R} \\
\left({ }^{\circ} \mathrm{C}\right)\end{array}$ & $\begin{array}{c}t_{c r} \\
(\mathrm{~min})\end{array}$ & $\begin{array}{c}X_{c r} \\
(\mathrm{~d} . \mathrm{b} .)\end{array}$ & $\begin{array}{c}X R_{c r} \\
(-)\end{array}$ & $\begin{array}{c}N_{w, c} \times 10^{3} \\
\left(\mathrm{~kg} \cdot \mathrm{m}^{-2} \cdot \mathrm{min}^{-1}\right)\end{array}$ \\
\hline 65 & $145 \pm 5$ & $1.477 \pm 0.117$ & $0.209 \pm 0.016$ & $52 \pm 3$ \\
80 & $95 \pm 5$ & $1.394 \pm 0.123$ & $0.205 \pm 0.009$ & $71 \pm 1$ \\
93 & $62 \pm 3$ & $1.539 \pm 0.162$ & $0.211 \pm 0.006$ & $99 \pm 4$ \\
\hline
\end{tabular}

\subsection{Influence of Shrinkage on Effective Moisture Diffusivity}

Diffusive models without and with shrinkage, Equations 4 and 10, were then used to estimate the effective diffusion coefficient of gel-coated seeds subjected to IR-drying. Table 2 shows the results obtained for the three IR source temperatures investigated in this study.

It can be verified that the values of diffusivity calculated without consideration of the shrinkage were higher than those obtained taking into account the phenomenon. For example, at $65^{\circ} \mathrm{C}$ the effective diffusivity of coated seeds without considering the shrinkage $\left(D_{\text {eff }}=12.1 \times\right.$ $10^{-11} \mathrm{~m}^{2} / \mathrm{min}$ ) was found to be $116 \%$ higher than that estimated taking into account the phenomenon $\left(D_{\text {eff }}^{*}=\right.$ $\left.5.51 \times 10^{-11} \mathrm{~m}^{2} / \mathrm{min}\right)$.

Neglecting shrinkage of coated seeds during IR drying leads, therefore, to an overestimation of the mass transfer by diffusion. The explanation is on the fact that particle shrinkage produces a variation in the distance required for the movement of water molecules, therefore making the effective diffusivity be overestimated when obtained from analytical solution of diffusion model without considering volume contraction. This finding agrees with results of previous reports on other products $[25,26]$.

The temperature dependency of the effective diffusivities estimated considering shrinkage was described by an Arrhenius-type relationship. The activation energy for infrared-dried coated seeds was found to be $33 \mathrm{~kJ} / \mathrm{mol}$, which is within the range of $12-110 \mathrm{~kJ} / \mathrm{mol}$ reported for several agricultural materials [15-18]. The Arrhenius factor was $5.161 \times 10^{-5} \mathrm{~m}^{2} / \mathrm{s}$, with $R^{2}$ of 0.9077 .

\subsection{Changes in Structural Properties}

The shrinkage-induced changes in structural properties of the gel-coated seeds were analyzed in terms of their apparent density. Typical results on particle density as a function of moisture content are shown in Figure 6 .

An explanation for the change in behavior of particle density during IR-drying may be ascribed to the distinct characteristics of reduction in mass and volume of the coated seed during moisture removal, which were pre-

Table 2. Values of effective moisture diffusivity estimated without and with consideration of shrinkage during IR drying of sorghum seeds encapsulated into agar gel.

\begin{tabular}{cccccccc}
\hline & \multicolumn{3}{c}{ Without shrinkage } & & \multicolumn{2}{c}{ With shrinkage } \\
\cline { 2 - 6 }$T_{\mathrm{IR}}\left({ }^{\circ} \mathrm{C}\right)$ & $D_{\text {eff }} \times 10^{11}\left(\mathrm{~m}^{2} / \mathrm{s}\right)$ & $R^{2}$ & RMSE & $D_{\text {eff }}^{*} \times 10^{11}\left(\mathrm{~m}^{2} / \mathrm{s}\right)$ & 0.9943 \\
\hline 65 & 7.57 & 0.9959 & 0.0066 & 4.55 & 0.0258 \\
80 & 12.1 & 0.9964 & 0.0051 & 5.51 & 0.9899 \\
93 & 20.7 & 0.9953 & 0.0050 & 11.3 & 0.9828 \\
\hline
\end{tabular}




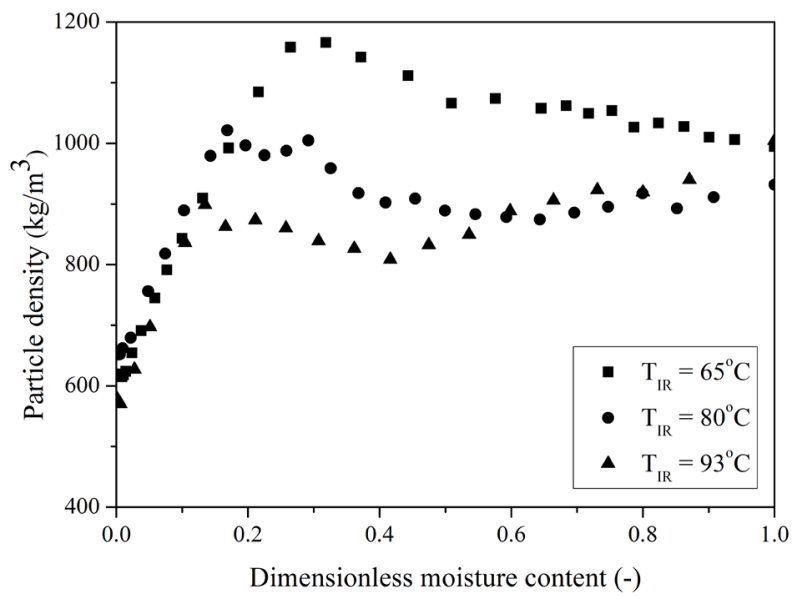

Figure 6. Particle density as a function of moisture content, for different IR source temperatures.

viously discussed.

In the whole range of temperatures investigated, coated seeds had its apparent density almost constant during the constant flux period, due to a reduction of both particle volume and amount of water evaporated in the same proportions. In the decreasing flux period as the volume contraction of particle is limited particle density steadily decreased with the loss of moisture. This result is an indicative that the pore formation within the gelbased coating structure begins at moisture contents below the critical moisture content. Similar behavior has been reported during IR drying of quince [34].

It can also be seen from Figure 6 that the IR drying temperature affected the apparent density of coated seeds. Apparent density of gel-coated seeds produced after IR drying at $65^{\circ} \mathrm{C}$ was about $17 \%$ higher than that obtained at $93^{\circ} \mathrm{C}$. The denser structure obtained at $65^{\circ} \mathrm{C}$ suggests that there was a reduction of pore volume within the gelbased coating structure as a result of a more uniform shrinkage during slow drying rate conditions. On the other hand, under rapid drying rate conditions, particle surface becomes fast unsaturated in moisture, so that low moisture content of this external surface may induce a rubber-glass transition and the formation of a porous outer rigid crust or shell that fixes the volume and hinders subsequent shrinkage of the still rubbery, inner part of the gel coating [29]. However, this contributes for replacement of evaporated water by air, thus leading to lower values of particle density.

The quality of the coated seeds produced at each temperature was evaluated in terms of the breakage percent in the artificial coating. Figure 7 shows the percent of breakage in coated particles versus the temperature employed in the drying process.

The percent of breakage ranged from $8 \%$ to $42 \%$ as the drying temperature increased from $65^{\circ} \mathrm{C}$ to $93^{\circ} \mathrm{C}$. This increase in cracking might be attributed to the

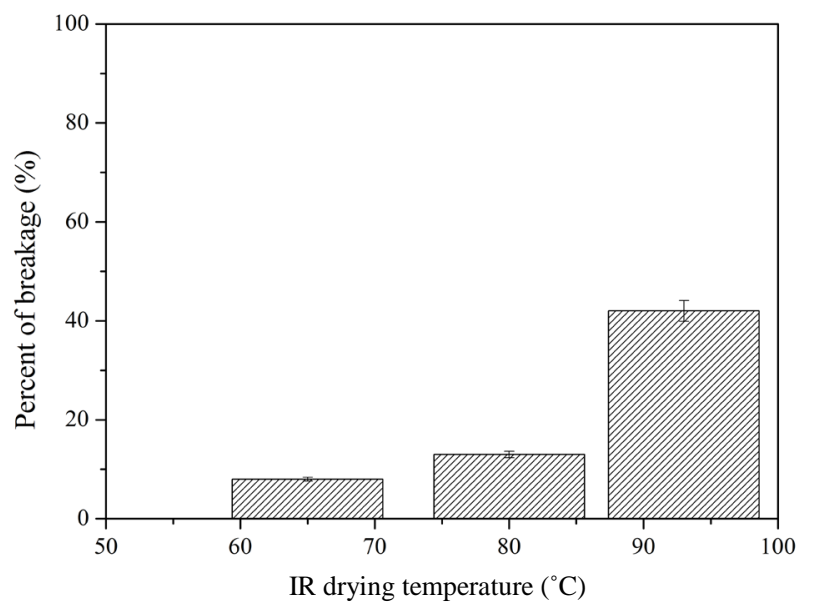

Figure 7. Percent of breakage in coated seeds during IR drying at different temperatures.

marked increase in water flux density as the IR heating temperature increased (Figure 5). Rapid drying rate conditions could have contributed to intense moisture gradients within the gel-based coating and, thus, to a higher state of stress in the gel-seed system, resulting in a high percent of breakage.

To produce gel-coated sorghum seeds with their noncracking structures mild drying conditions must therefore be applied. Modifying the composition of the coating matrix is another alternative, in order to retard its loss of elasticity during drying, thus increasing the resistance to breakage.

\section{Conclusions}

The analysis of drying kinetics based on both evolutions of solid temperature and water flux density taking into account the changes in mass exchange area is essential to obtain an accurate interpretation of mass transfer in IRdrying of shrinking particles like sorghum seeds encapsulated into gel-based polymeric matrix.

The IR drying behavior of gel-coated seeds was characterized by the presence of three periods of drying: warming-up, constant flux and one falling flux period.

Gel-coated seeds subjected to drying using infrared radiation had significant shrinkage, which occurred mainly during the constant flux period. In this stage of drying, the particle density was almost constant with reduction in moisture content. The decrease in apparent density during the falling moisture flux period evidenced a volume contraction of particles lower than the amount of removed water.

Changes in surface area and volume of coated seeds were found to be affected by IR source temperature during the constant flux period, when an increase in temperature reduced particle shrinkage. At the final stage of drying, the aforementioned changes were dependent only 
on the average moisture content of particles.

Neglecting the shrinkage of the particles led to a significant overestimation of the effective moisture diffusivity and thus of the energy required for drying the material.

IR drying temperature significantly affected apparent density of gel-coated seeds and this influence is closely related to the rates at which both the volume shrinkage and drying occur. Particles dried under rapid drying rate conditions had the lowest apparent density values.

Percent of cracks in gel-coated seeds produced by high-temperature IR drying was greater than that obtained at low-temperature IR drying. Thus, for the polymer coating used to encapsulate sorghum seeds, only mild drying rate conditions should be applied to minimize cracking under moisture removal, thus preserving structural integrity of the gel-seed system.

\section{Acknowledgements}

The authors are grateful to CNPq (National Council for Research) and FAPITEC (Foundation for Research Support of Sergipe State) for the financial support received.

\section{REFERENCES}

[1] G. Koçar and N. Civaş, "An Overview of Biofuels from Energy Crops: Current Status and Future Prospects," Renewable and Sustainable Energy Reviews, Vol. 28, 2013, pp. 900-916. http://dx.doi.org/10.1016/j.rser.2013.08.022

[2] G. G. Cordeiro, G. M. de Resende, J. R. Pereira and N. D. Costa, "Effect of Saline Water and Soil Conditioner on Sugar Beet Yield in the Brazilian Semi-Arid Region," Horticultura Brasileira, Vol. 17, No. 1, 1999, pp. 39-41. http://dx.doi.org/10.1590/S0102-05361999000100010

[3] M. D. Kaya, G. Okc, M. Atak, Y. Çıkılı and O. Kolsarıcı, "Seed Treatments to Overcome Salt and Drought Stress during Germination in Sunflower (Helianthus annuus L.)," European Journal of Agronomy, Vol. 24, No. 4, 2006, pp. 291-296. http://dx.doi.org/10.1016/j.eja.2005.08.001

[4] A. G. Taylor, P. S. Allen and M. K. Misra, "Seed Enhancements," Seed Science Research, Vol. 8, No. 21, 1998, pp. 245-256.

[5] M. M. Prado, M. M. Ferreira and D. J. M. Sartori, "Drying of Seeds and Gels,” Drying Technology, Vol. 24, No. 3, 2006, pp. 281-292. http://dx.doi.org/10.1080/07373930600564514

[6] S. Sarrocco, R. Raeta and G. Vannacci, "Seeds Encapsulation in Calcium Alginate Pellets," Seed Science and Technology, Vol. 32, No. 3, 2004, pp. 649-661.

[7] J. Kajtna, U. Sebenik, M. Krajnc and J. Golob, "IR Drying of Water-Based Acrylic PSA Adhesives,” Drying Technology, Vol. 26, No. 3, 2008, pp. 323-333. http://dx.doi.org/10.1080/07373930801898059

[8] G. S. V. Raghavan, T. J. Rennie, P. S. Sunjka, V. Orsat, W. Phaphuangwittayakul and P. Terdtoon, "Overview of
New Techniques for Drying Biological Materials with Emphasis on Energy Aspects,” Brazilian Journal of Chemical Engineering, Vol. 22, No. 2, 2005, pp. 195-201. http://dx.doi.org/10.1590/S0104-66322005000200005

[9] H. Kocabiyik and D. Tezer, "Drying of Carrot Slices Using Infrared Radiation,” International Journal of Food Science and Technology, Vol. 44, No. 5, 2009, pp. 953959. http://dx.doi.org/10.1111/j.1365-2621.2008.01767.x

[10] J. Seyed-Yagoobi and J. W. Wirtz, "An Experimental Study of Gas-Fired Infrared Drying of Paper,” Drying Technology, Vol. 19, No. 6, 2001, pp. 1099-1112. http://dx.doi.org/10.1081/DRT-100104807

[11] S. Le Person, J. R. Puiggali, M. Baron and M. Roques, "Near Infrared Drying of Pharmaceutical Thin Films: Experimental Analysis of Internal Mass Transport," Chemical Engineering and Processing, Vol. 37, No. 3, 1998, pp. 257-263. http://dx.doi.org/10.1016/S0255-2701(98)00032-4

[12] D. Blanc, P. Laurent, J. F. Gerard and J. Andrieu, "Experimental Infrared Drying Study of a Model Water-Based Epoxy-Amine Painting Coated on Iron Support," Drying Technology, Vol. 15, No. 6-8, 1997, pp. 1787-179 9.

[13] S. B. Pawar, P. S. R. Kumar, A. S. Mujumdar and B. N. Thorat, "Infrared-Convective Drying of Organic Pigments," Drying Technology, Vol. 26, No. 3, 2008, pp. 315-322. http://dx.doi.org/10.1080/07373930801898042

[14] P. Glouannec, P. Salagnac, H. Guézenoc and N. Allanic, "Experimental Study of Infrared-Convective Drying of Hydrous Ferrous Sulphate,” Powder Technology, Vol. 187, No. 3, 2008, pp. 280-288. http://dx.doi.org/10.1016/j.powtec.2008.03.007

[15] A. R. Celma, S. Rojas and F. Lopez-Rodríguez, "Mathematical Modelling of Thin-Layer Infrared Drying of Wet Olive Husk," Chemical Engineering and Processing, Vol. 47, No. 9-10, 2008, pp. 1810-1818. http://dx.doi.org/10.1016/j.cep.2007.10.003

[16] N. Boudhrioua, N. Bahloul, I. B. Ben and N. K. Slimen, "Comparison on the Total Phenol Contents and the Color of Fresh and Infrared Dried Olive Leaves," Industrial Crops and Products, Vol. 2, No. 2-3, 2009, pp. 412-419. http://dx.doi.org/10.1016/j.indcrop.2008.08.001

[17] I. Das, S. K. Das and S. Bal, "Drying Kinetics of High Moisture Paddy Undergoing Vibration-Assisted Infrared (IR) Drying," Journal of Food Engineering, Vol. 95, No. 1, 2009, pp. 166-171. http://dx.doi.org/10.1016/j.jfoodeng.2009.04.028

[18] C. Niamnuy, M. Nachaisin, N. Poomsa-ad and S. Devahastin, "Kinetics Modeling of Drying and Conversion/ Degradation of Isoflavones during Infrared Drying of Soybean," Food Chemistry, Vol. 133, No. 3, 2012, pp. 946952. http://dx.doi.org/10.1016/j.foodchem.2012.02.010

[19] S. Eichler, O. Ramon and I. Ladyzhinski, "Collapse Processes in Shrinkage of Hydrophilic Gels during Dehydration,” Food Research International, Vol. 30, No. 9, 1997, pp. 719-726. http://dx.doi.org/10.1016/S0963-9969(98)00028-3

[20] G. Hashemi, D. Mowla and M. Kazemeini, "Moisture Diffusivity and Shrinkage of Broad Beans during Bulk Drying in an Inert Medium Fluidized Bed Dryer Assisted 
by Dielectric Heating,” Journal of Food Engineering, Vol. 92, No. 3, 2009, pp. 331-338. http://dx.doi.org/10.1016/j.jfoodeng.2008.12.004

[21] I. Bialobrzewski, M. Zielínska, A. S. Mujumdar and M. Makowski, "Heat and Mass Transfer during Drying of a Bed of Shrinking Particles-Simulation for Carrot Cubes Dried in a Spout-Fluidized-Bed Drier," International Journal of Heat and Mass Transfer, Vol. 51, No. 19, 2008, pp. 4704-4716.

http://dx.doi.org/10.1016/j.ijheatmasstransfer.2008.02.031

[22] Committee on Food Chemicals Codex, "Food Chemical Codex," 5th Edition, National Academies Press, Washington DC, 2004.

[23] O. Fasina, B. Tyler, M. Pickard, G. Zheng and N. Wang, "Effect of Infrared Heating on the Properties of Legume Seeds," International Journal of Food Science and Technology, Vol. 36, No. 1, 2001, pp. 79-90. http://dx.doi.org/10.1046/j.1365-2621.2001.00420.x

[24] J. Crank, "The Mathematics of Diffusion," 2nd Edition, Clarendon Press, Oxford, London, 1975.

[25] B. A. Souraki and D. Mowla, “Axial and Radial Moisture Diffusivity in Cylindrical Fresh Green Beans in a Fluidized Bed Dryer with Energy Carrier: Modeling with and without Shrinkage," Journal of Food Engineering, Vol. 88, No. 1, 2008, pp. 9-19. http://dx.doi.org/10.1016/j.jfoodeng.2007.05.013

[26] A. Arévalo-Pinedo and F. E. X. Murr, "Kinetics of Vacuum Drying of Pumpkin (Cucurbita maxima): Modeling with Shrinkage," Journal of Food Engineering, Vol. 76, No. 4, 2006, pp. 562-567. http://dx.doi.org/10.1016/j.jfoodeng.2005.06.003

[27] R. Fletcher, "Pratical Methods of Optimization," 2nd Edition, Wiley, London, 2001.
[28] C. Ratti, "Shrinkage during Drying of Foodstuffs," Journal of Food Engineering, Vol. 23, No. 1, 1994, pp. 91105. http://dx.doi.org/10.1016/0260-8774(94)90125-2

[29] L. Mayor and A. M. Sereno, "Modelling Shrinkage during Convective Drying of Food Materials: A Review," Journal of Food Engineering, Vol. 61, No. 3, 2004, pp. 373-386. http://dx.doi.org/10.1016/S0260-8774(03)00144-4

[30] S. Pabis and M. Jaros, "The First Period of Convection of Vegetables and the Effect of Shape-Dependent Shrinkage,” Biosystems Engineering, Vol. 81, No. 2, 2002, pp. 201211. http://dx.doi.org/10.1006/bioe.2001.0015

[31] P. Perré and B. May, "The Importance of Considering Exchange Surface Area Reduction to Exhibit a Constant Drying Flux Period in Foodstuffs," Journal of Food Engineering, Vol. 54, No. 4, 2002, pp. 271-282. http://dx.doi.org/10.1016/S0260-8774(01)00213-8

[32] G. P. Sharma, R. C. Verma and P. Pathare, "Mathematical Modeling of Infrared Radiation Thin Layer Drying of Onion Slices,” Journal of Food Engineering, Vol. 71, No. 3, 2005, pp. 282-286. http://dx.doi.org/10.1016/j.jfoodeng.2005.02.010

[33] A. K. Datta and H. Ni, "Infrared and Hot-Air-Assisted Microwave Heating of Foods for Control of Surface Moisture,” Journal of Food Engineering, Vol. 51, No. 4, 2002, pp. 355-364.

http://dx.doi.org/10.1016/S0260-8774(01)00079-6

[34] I. Koç, R. I. Eren and F. K. Ertekin, "Modelling Bulk Density, Porosity and Shrinkage of Quince During Drying: The Effect of Drying Method," Journal of Food Engineering, Vol. 85, No. 3, 2008, pp. 340-349. http://dx.doi.org/10.1016/j.jfoodeng.2007.07.030 